\title{
ANÁLISE EXERGÉTICA DE UM CICLO RANKINE EM CONDIÇÕES SUPERCRÍTICAS UTILIZANDO A EQUAÇÃO DE ESTADO DE PENG-ROBINSON E FERRAMENTAS DO MATLAB (GUI)
}

\author{
J. V. L. R. FERRAZ, P. F. ARCE CASTILLO \\ Universidade de São Paulo, Escola de Engenharia de Lorena, Departamento de \\ Engenharia Química \\ E-mail para contato: joao.vflr@alunos.eel.usp.br
}

\begin{abstract}
RESUMO - O presente trabalho tem como objetivo analisar, de um ponto de vista exergético, ciclos de potência Rankine em condições de operação sub e supercrítica, focando tal análise na contribuição exergética de cada corrente, de forma que é calculada a diferença de propriedades termodinâmicas de entrada e saída entre cada equipamento. Para o cálculo das propriedades termodinâmicas (entalpia, entropia e exergia), foram utilizadas as funções de partida, obtidas a partir da Equação de Estado de Peng-Robinson e fazendo uso de ferramentas gráficas do MatLab (GUI). Os resultados do rendimento termodinâmico e da exergia de cada corrente indicam que há uma melhora significativa no rendimento do ciclo quando são usados fluidos supercríticos, o que torna interessante estudos posteriores que visem melhorar a eficiência dos equipamentos industriais ou modificar o fluido de operação, de forma a tornar o desempenho geral de geração de potência ainda melhor.
\end{abstract}

\section{INTRODUÇÃO}

\subsection{Exergia e análise exergética}

A busca por novas maneiras de gerar energia com qualidade é constante para o ser humano. Um dos princípios mais famosos da física dita que "toda energia se conserva". Entretanto, uma vez que tal verdade é posta à prova, pode-se verificar que, embora o balanço seja realmente perfeito, parte da energia é dissipada para o ambiente, o que causa perdas na capacidade de geração de trabalho efetivo. Isso porque um segundo princípio, também muito famoso, dita que "a entropia de um sistema tende sempre a aumentar". Dessa maneira, foi necessário criar um terceiro conceito, capaz de unir as duas leis supracitadas. Assim surgiu o conceito de exergia. Segundo Dincer e Rosen (2007), a exergia é o máximo trabalho que pode ser executado por um sistema e uma vizinhança devidamente definida. Dessa maneira, trata-se, em última instância, de uma união entre as duas leis, uma forma de medir a "qualidade" da energia. Basicamente, há quatro formas de exergia: cinética, potencial, química e mecânica. Uma nova tendência ganha cada vez mais força na engenharia moderna: a análise exergética. Ela é extremamente mais completa na realização de uma análise da autonomia de diversos ciclos termodinâmicos e máquinas térmicas, pois permite apontar quais etapas do processo estão apresentando 
um pior desempenho, redirecionando os esforços para solucionar os problemas de tal etapa primeiro, o que garante uma otimização dos resultados finais do processo.

\subsection{Ciclos termodinâmicos}

Para uma análise exergética, os ciclos termodinâmicos são interessantíssimos: não só se pode avaliar a eficiência de cada equipamento, como também é possível estudar a do fluido de operação. Um ciclo Rankine, conforme mostrado na Figura 1, opera, normalmente, utilizando água, e é composto por 4 equipamentos: bomba, caldeira, turbina e condensador.

Figura 1 - Configuração de um ciclo Rankine

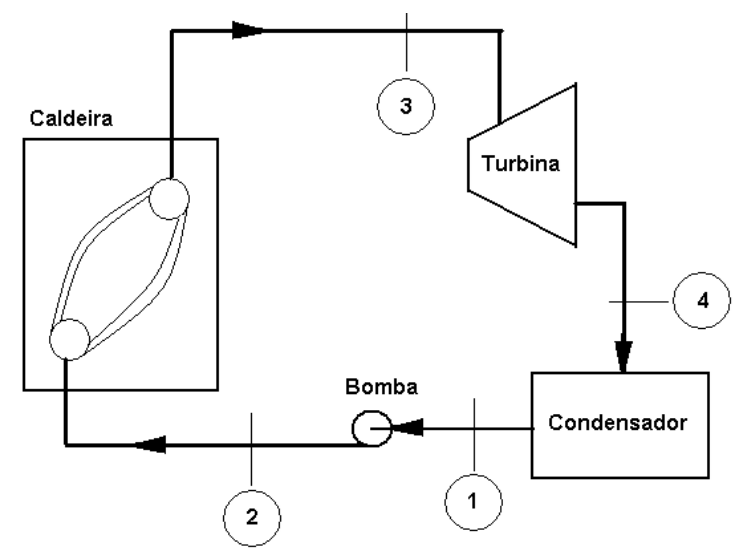

Diversas modificações podem ser realizadas nesses ciclos, desde acréscimo de bombas e turbinas até múltiplas passagens do fluido de operação pela caldeira. Todas elas visam aumentar o rendimento geral do ciclo $(\eta)$, que é calculado a partir da Equação 1.

$$
\eta=\frac{W_{t u r b}-W_{b o m b}}{Q_{h}}
$$

\subsection{Fluidos supercríticos}

Os primeiros a observarem o efeito do aumento das condições de pressão e temperatura de um fluido foram Hannay e Hogarth (1879), embora, à época, as possibilidades de usos práticos fossem extremamente baixas, pois os equipamentos eram extremamente limitados no que diz respeito a suportar condições elevadas de temperatura e pressão. Diz-se que um fluido atingiu o estado supercrítico quando suas condições de temperatura e pressão ultrapassam as do ponto crítico, conforme mostra a Figura 2 (S: sólido, L: líquido, V: vapor, G: gás, P: pressão, T: temperatura, Pc: pressão crítica, Tc: temperatura crítica).

Entretanto, apesar de usualmente definido a partir de diagramas de fases, o conceito gráfico tem pouca importância prática, uma vez que a passagem do estado gasoso ou líquido para o supercrítico ocorre de uma forma contínua. Neste trabalho, condições sub e supercríticas de operação são propostas para um ciclo Rankine, de forma 
que é realizado o balanço geral de tais ciclos. Para otimizar os cálculos, será utilizado um programa computacional, representado na Figura 3, desenvolvido no software MatLab, utilizando interfaces gráficas de usuário (GUI). As condições de operação serão comparadas, de forma a ilustrar os ganhos reais, por meio de uma análise exergética dos ciclos, buscando o equipamento com o pior aproveitamento exergético.

Figura 2 - Representação da região supercrítica

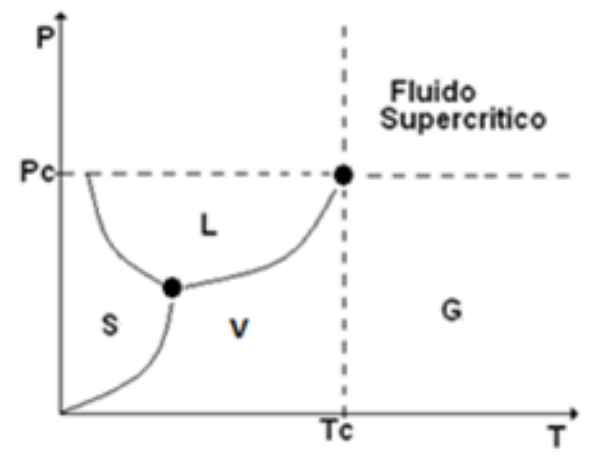

Figura 3 - Interface gráfica desenvolvida em MatLab

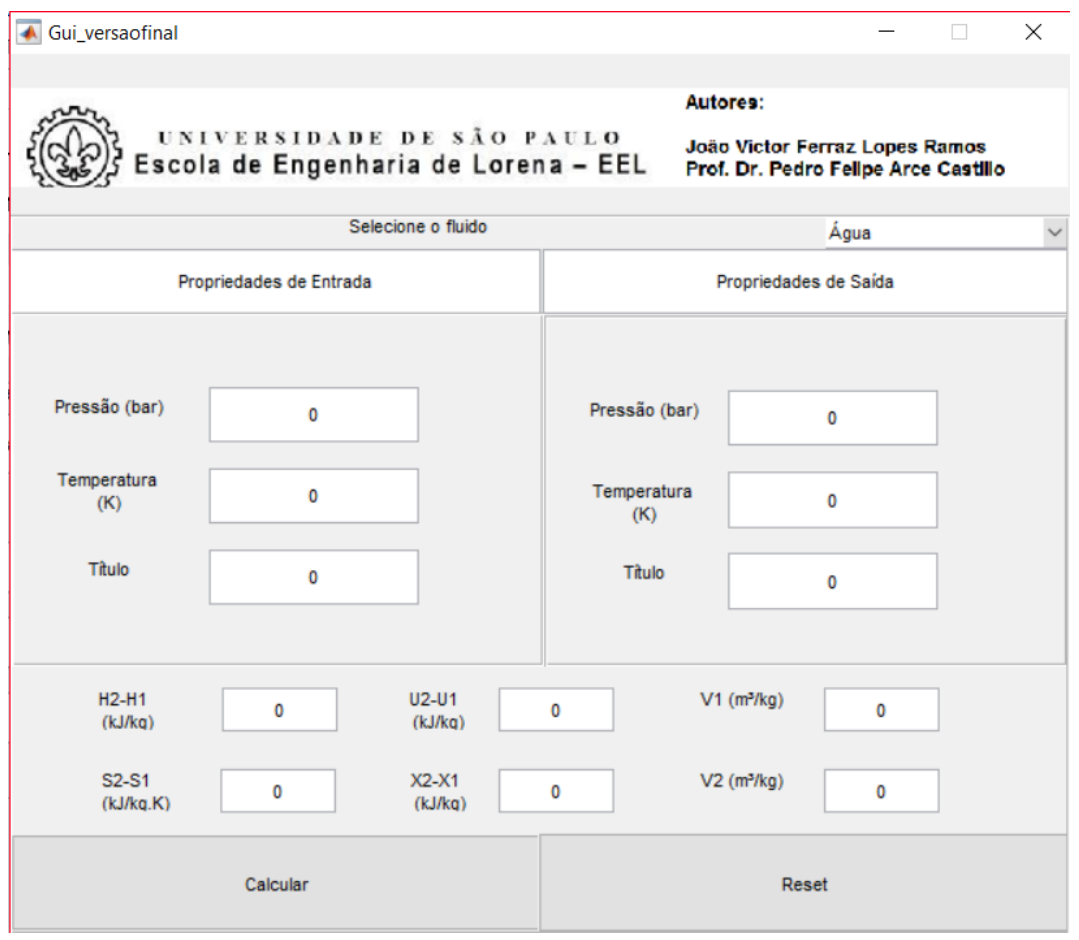

\section{MATERIAIS E MÉTODOS}

\subsection{Dados operacionais dos ciclos Rankine para análise}

As condições de operação do ciclo estão descritas nas Tabelas 1 e 2 . 


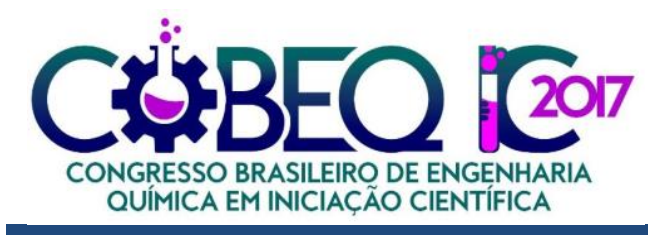
XII Congresso Brasileiro de Engenharia
Química em Iniciação Científica
UFSCar - São Carlos - SP
16 a 19 de Julho de 2017

Tabela 1 - Condições de operação usual

\begin{tabular}{|c|c|c|c|c|}
\hline Corrente & $\begin{array}{c}\text { Pressão } \\
\text { (MPa) }\end{array}$ & $\begin{array}{c}\text { Temperatura } \\
(\mathbf{K})\end{array}$ & $\begin{array}{c}\text { Eficiência } \\
\text { Isentrópica }\end{array}$ & Estado Termodinâmico \\
\hline $\mathbf{1}$ & 0,01 & 318,96 & \multirow{2}{*}{$85,0 \%$} & Líquido Saturado \\
\hline $\mathbf{2}$ & 15,0 & 320,11 & & Líquido Comprimido \\
\hline $\mathbf{3}$ & 15,0 & 623,15 & \multirow{2}{*}{$85,0 \%$} & Vapor Superaquecido \\
\hline $\mathbf{4}$ & 0,01 & 318,96 & & Mistura Saturada (x =0,7000) \\
\hline
\end{tabular}

Tabela 2 - Condições de operação supercríticas

\begin{tabular}{|c|c|c|c|c|}
\hline Corrente & $\begin{array}{c}\text { Pressão } \\
\text { (MPa) }\end{array}$ & $\begin{array}{c}\text { Temperatura } \\
(\mathbf{K})\end{array}$ & $\begin{array}{c}\text { Eficiência } \\
\text { Isentrópica }\end{array}$ & Estado Termodinâmico \\
\hline $\mathbf{1}$ & 0,01 & 318,96 & \multirow{2}{*}{$85,0 \%$} & Líquido Saturado \\
\hline $\mathbf{2}$ & 50,0 & 322,83 & & Líquido Comprimido \\
\hline $\mathbf{3}$ & 50,0 & 923,15 & \multirow{2}{*}{$85,0 \%$} & Fluido Supercrítico \\
\hline $\mathbf{4}$ & 0,01 & 318,96 & & Mistura Saturada (x $=0,8141)$ \\
\hline
\end{tabular}

\subsection{Funções de partida obtidas a partir da equação cúbica de estado de Peng-Robinson}

As funções de partida consistem em um "artifício" utilizado para o cálculo de propriedades termodinâmicas utilizando uma equação de estado. Partindo do princípio que, para ir de um estado termodinâmico a outro, o caminho utilizado não interfere, as equações de estado podem ser desenvolvidas, de forma que são capazes de calcular diversas propriedades termodinâmicas. Elas calculam a diferença de propriedades entre um estado real e um ideal. Essa lógica pode ser melhor representada pela Figura 4 (P: pressão, $\mathrm{T}$ : temperatura, $\Delta \mathrm{P}$ : variação de pressão, $\Delta \mathrm{T}$ : variação de temperatura, ig: estado de gás ideal, $\bullet$ : estado $1, \bullet$ : estado 2.

Figura 4 - Lógica das funções de partida

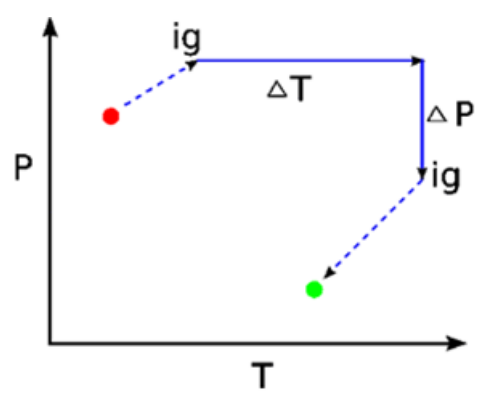

As funções de partida de entalpia e entropia obtidas a partir da equação cúbica de estado de Peng-Robinson (1976) são representadas pelas Equações 2 e 3: 


$$
\begin{gathered}
\frac{H_{T, P}-H_{T, P}^{i g}}{R T}=(Z-1)-\frac{A}{B \sqrt{8}}\left(1+\frac{m \sqrt{T r}}{\sqrt{\alpha}}\right) \ln \left[\frac{Z+(1+\sqrt{2}) B}{Z+(1-\sqrt{2}) B}\right] \\
\frac{S_{T, P}-S_{T, P}^{\text {ideal }}}{R}=\ln (Z-B)-\frac{A}{B \sqrt{8}} \frac{m \sqrt{T r}}{\sqrt{\alpha}} \ln \left[\frac{Z+(1+\sqrt{2}) B}{Z+(1-\sqrt{2}) B}\right]
\end{gathered}
$$

Assim, para calcular a variação total de uma propriedade termodinâmica, basta aplicar as funções de partida, de forma a ir do estado real 1 para o estado de gás ideal 1. As Equações 4 e 5 calculam a diferença entre os estados ideais 1 e 2:

$$
\begin{aligned}
& H_{2}^{i g}-H_{1}^{i g}=k_{1}(\Delta T)+\frac{k_{2}}{2}\left(T_{2}^{2}-T_{1}^{2}\right)+\frac{k_{3}}{3}\left(T_{2}^{3}-T_{1}^{3}\right)+\frac{k_{4}}{4}\left(T_{2}^{4}-T_{1}^{4}\right) \\
& S_{2}^{i g}-S_{1}^{i g}=k_{1} \ln \left(\frac{T_{2}}{T_{1}}\right)+k_{2}\left(T_{2}-T_{1}\right)+\frac{k_{3}}{2}\left(T_{2}^{2}-T_{1}^{2}\right)+\frac{k_{4}}{3}\left(T_{2}^{3}-T_{1}^{3}\right) \\
& -R \ln \left(\frac{P_{2}}{P_{1}}\right)
\end{aligned}
$$

Finalmente, as Equações 2 e 3 são novamente aplicadas, dessa vez na passagem do estado ideal 2 e real 2. Assim, a diferença de propriedades entre os estados reais 1 e 2 é finalmente determinada pelo somatório dos termos anteriores.

\section{RESULTADOS}

Os resultados obtidos para a exergia $(\mathrm{X})$ utilizando tabelas termodinâmicas e o software, bem como os erros relativos da exergia podem ser visualizados nas Tabelas $3 \mathrm{e}$ 4.

Tabela 3 - Resultados obtidos para o caso subcrítico

\begin{tabular}{|c|c|c|c|}
\hline & \multicolumn{3}{|c|}{ Resultados obtidos para o caso subcrítico } \\
\hline \multirow{2}{*}{ Equipamento } & Tabelas & Software & \multirow{2}{*}{ Erros relativos (\%) } \\
\cline { 2 - 3 } & $\Delta \mathrm{X}(\mathrm{kJ} / \mathrm{kg})$ & $\Delta \mathrm{X}(\mathrm{kJ} / \mathrm{kg})$ & \\
\hline Bomba & 15,33 & 17,84 & $16,37 \%$ \\
\hline Caldeira & 1056,14 & 1329,53 & $25,89 \%$ \\
\hline Turbina & $-688,77$ & $-1007,89$ & $46,33 \%$ \\
\hline Condensador & $-110,23$ & $-117,61$ & $6,70 \%$ \\
\hline
\end{tabular}

Ao comparar os resultados obtidos, alguns fatores extremamente interessantes merecem destaques. Vale observar que a equação de Peng-Robinson apresenta algumas irregularidades na determinação de propriedades termodinâmicas, de forma que pode não ser um método muito confiável. Sobre a questão exergética, há um ganho visível quando o fluido de operação se encontra em estado supercrítico na caldeira. 
Tabela 4 - Resultados obtidos para o caso supercrítico

\begin{tabular}{|c|c|c|c|}
\hline & \multicolumn{3}{|c|}{ Resultados obtidos para o caso supercrítico } \\
\hline \multirow{2}{*}{ Equipamento } & Tabelas & Software & \multirow{2}{*}{ Erros relativos $(\%)$} \\
\cline { 2 - 4 } & $\Delta \mathrm{X}(\mathrm{kJ} / \mathrm{kg})$ & $\Delta \mathrm{X}(\mathrm{kJ} / \mathrm{kg})$ & \\
\hline Bomba & 50,63 & 59,44 & $17,40 \%$ \\
\hline Caldeira & 1594,36 & 1772,53 & $11,18 \%$ \\
\hline Turbina & $-1086,77$ & $-1566,36$ & $44,13 \%$ \\
\hline Condensador & $-127,76$ & $-136,80$ & $7,08 \%$ \\
\hline
\end{tabular}

Quanto ao equipamento com a menor capacidade de aproveitamento de energia, trata-se da caldeira. Almeida (2011) propõe uma análise à luz da termodinâmica, de maneira que, para um ciclo Rankine, a fonte quente é o fluido utilizado no aquecimento do líquido comprimido. A fonte fria, por sua vez, é representada pelo condensador, sendo o ciclo de vapor a turbina, que transforma o calor em trabalho.

\section{CONCLUSÕES}

A análise exergética aponta claros ganhos no uso de fluidos supercríticos para geração de energia, de maneira que uma análise de viabilidade econômica de implantação de tais fluidos deve ser realizada, como forma de avaliar a questão de custos. Quanto às funções de partida, é interessante modificar a EdE Peng-Robinson ou buscar outras equações de estado, uma vez que alguns resultados destoaram de formas gritantes da realidade (chegando a ultrapassar o erro relativo de $40 \%$ ).

\section{REFERÊNCIAS}

ALMEIDA, A. F. Análise exergética dos ciclos rankine e combinado com gaseificação integrada operados a carvão mineral. 2011. 100 f. Dissertação (Mestrado em Engenharia Mecânica) - Programa de Pós-graduação em Engenharia Mecânica (COPPE), Universidade Federal do Rio de Janeiro, Rio de Janeiro, 2011.

DINCER, I.; ROSEN, M. A. Exergy: Energy, Environment and Sustainable Development. Elsevier, 2007.

HANNAY, J. B.; HOGARTH, J.; On the Solubility of Solids in Gases. Londres, Royal Society of London, 1879

PENG, D.Y.; ROBINSON, D.B.; A New Two-Constant Equation of State. Ind. Eng. Quim. Fundamen., 1976. p 59-64.

\section{AGRADECIMENTOS}

J.V.L.R. Ferraz agradece à Escola de Engenharia de Lorena (EEL) da Universidade de São Paulo (USP) pela oportunidade de realizar sua primeira Iniciação Científica e P.F. Arce Castillo agradece à Fundação de Amparo à Pesquisa do Estado de São Paulo (FAPESP) através do processo 2015/05155-8 pelo auxílio financeiro à pesquisa. 